\title{
Is a Responsive Default Mode Network Required for Successful Working Memory Task Performance?
}

\author{
Marta Čeko, ${ }^{1}$ John L. Gracely, ${ }^{1}$ Mary-Ann Fitzcharles, ${ }^{5}$ DDavid A. Seminowicz, ${ }^{6}$ Petra Schweinhardt, ${ }^{2,3,4}$ \\ and M. Catherine Bushnell ${ }^{1}$ \\ ${ }^{1}$ National Center for Complementary and Integrative Health, National Institutes of Health, Bethesda, Maryland 20892, ${ }^{2}$ Alan Edwards Centre for Research \\ on Pain, ${ }^{3}$ Dentistry, ${ }^{4}$ Neurology and Neurosurgery, and ${ }^{5}$ Rheumatology, McGill University, Montreal, Quebec H3A 0G4, Canada, and ${ }^{6}$ Department of Neural \\ and Pain Sciences, School of Dentistry, University of Maryland, Baltimore, Maryland 21201
}

In studies of cognitive processing using tasks with externally directed attention, regions showing increased (external-task-positive) and decreased or "negative" [default-mode network (DMN)] fMRI responses during task performance are dynamically responsive to increasing task difficulty. Responsiveness (modulation of fMRI signal by increasing load) has been linked directly to successful cognitive task performance in external-task-positive regions but not in DMN regions. To investigate whether a responsive DMN is required for successful cognitive performance, we compared healthy human subjects $(n=23)$ with individuals shown to have decreased DMN engagement (chronic pain patients, $n=28$ ). Subjects performed a multilevel working-memory task ( $N$-back) during fMRI. If a responsive DMN is required for successful performance, patients having reduced DMN responsiveness should show worsened performance; if performance is not reduced, their brains should show compensatory activation in external-task-positive regions or elsewhere. All subjects showed decreased accuracy and increased reaction times with increasing task level, with no significant group differences on either measure at any level. Patients had significantly reduced negative fMRI response (deactivation) of DMN regions (posterior cingulate/precuneus, medial prefrontal cortex). Controls showed expected modulation of DMN deactivation with increasing task difficulty. Patients showed significantly reduced modulation of DMN deactivation by task difficulty, despite their successful task performance. We found no evidence of compensatory neural recruitment in external-task-positive regions or elsewhere. Individual responsiveness of the external-task-positive ventrolateral prefrontal cortex, but not of DMN regions, correlated with task accuracy. These findings suggest that a responsive DMN may not be required for successful cognitive performance; a responsive external-task-positive network may be sufficient.

Key words: chronic pain; default mode; external-task-positive; FMRI; task performance; working memory

Significance Statement

We studied the relationship between responsiveness of the brain to increasing task demand and successful cognitive performance, using chronic pain patients as a probe. fMRI working memory studies show that two main cognitive networks ["external-task positive" and "default-mode network" (DMN)] are responsive to increasing task difficulty. The responsiveness of both of these brain networks is suggested to be required for successful task performance. The responsiveness of external-task-positive regions has been linked directly to successful cognitive task performance, as we also show here. However, pain patients show decreased engagement and responsiveness of the DMN but can perform a working memory task as well as healthy subjects, without demonstrable compensatory neural recruitment. Therefore, a responsive DMN might not be needed for successful cognitive performance.

\section{Introduction}

fMRI studies of cognitive processing in healthy subjects reveal two main sets of regions with opposite neural recruitment during performance of tasks requiring externally directed attention ("external tasks"), such as working memory (Fox et al., 2005; Fransson, 2005). Regions that are "external-task-positive" show increased fMRI activity and include lateral prefrontal, midcingulate, and posterior parietal cortical areas. 
Regions of the so-called default mode network (DMN), including the posterior cingulate (PCC), precuneus, medial prefrontal (MPFC), and temporal cortical areas, show decreased $\mathrm{fMRI}$ response (i.e., negative $\mathrm{fMRI}$ signal or "deactivation") during task performance. DMN regions are active at rest and commonly during cognitive processes requiring internally directed attention, such as episodic memory, self-generated thought, mind wandering, rumination, or reflection (Gusnard et al., 2001; Raichle et al., 2001; Christoff et al., 2009; Kross et al., 2009; Andrews-Hanna et al., 2010b; Kucyi et al., 2013).

When task difficulty varies (e.g., in a multilevel working memory task), more activation is observed typically in externaltask-positive regions with increasing task difficulty (Barch et al., 1997; Braver et al., 1997; Tomasi et al., 2007). This modulation of fMRI activity by task load (i.e., responsiveness to increasing task load) in externaltask-positive regions has been linked directly to task performance, so that those subjects with more responsive externaltask-positive regions to increasing task load had more accurate performance at the most difficult level of the task (Nagel et al., 2009, 2011; Burzynska et al., 2011).

With increasing task difficulty, the DMN typically shows more pronounced deactivation (Shulman et al., 1997; McKiernan et al., 2003; Todd et al., 2005; Mayer et al., 2010), interpreted to reflect a necessary reallocation of limited cognitive resources toward external-task-positive processes (Shulman et al., 1997; McKiernan et al., 2006; Mayer et al., 2010). It has been suggested that the DMN is not just deactivated during external tasks but that the modulation of deactivation by task load in the DMN (i.e., responsiveness to increasing task load) facilitates successful cognitive performance (Sambataro et al., 2010; Anticevic et al., 2012a; Metzak et al., 2012). However, whereas the responsiveness of external-taskpositive regions to increasing task load has been linked directly to successful cognitive task performance, it has not been established whether the responsiveness of DMN to increasing task load is required for the ability to successfully perform the task.

To investigate whether a responsive DMN is required for successful working memory performance, we compared healthy controls who show typical recruitment and responsiveness (modulation by task load) of cognitive networks with individuals shown to have decreased DMN deactivation, i.e., chronic pain patients (Baliki et al., 2008; Seminowicz et al., 2011; WeissmanFogel et al., 2011). Groups performed a multilevel working memory task ( $N$-back) while undergoing fMRI. We compared task-related recruitment of external-task-positive regions and the DMN, and their modulation by cognitive load.

We reasoned that, if a responsive DMN is required for successful task performance, one of the following conditions would be met, given comparable intellectual capacity and education

The authors declare no competing financial interests.

Correspondence should be addressed to M. Catherine Bushnell, Building 35A, Room 1D822, 35A Convent Drive, Bethesda, MD 20892. E-mail: mary.bushnell@nih.gov.

DOI:10.1523/JNEUROSCI.0264-15.2015

Copyright $\odot 2015$ the authors $\quad 0270-6474 / 15 / 3511596-11 \$ 15.00 / 0$ level across groups: (1) pain patients perform worse and show less deactivation and less modulation by increasing cognitive load (i.e., responsiveness) of the DMN; (2) groups have similar task performance together with similar deactivation and responsiveness of the DMN; or (3) groups have similar task performance with patients showing impaired deactivation and responsiveness of the DMN, with compensatory recruitment of external-taskpositive regions to maintain performance (Fig. 1). Alternatively, we would conclude that a responsive DMN is not required for successful working memory performance.

\section{Materials and Methods}

\section{Subjects}

In this study, we compared 28 female chronic pain patients diagnosed with fibromyalgia (mean age \pm SD, $48.7 \pm 7.8$ years; range, $29-60$ years) and 28 individually age-matched ( \pm 3 years) healthy females ( $48.8 \pm 7.7$ years; range, $30-63$ years; $p=0.973$ ). The data reported here are part of a larger study. Previously, we reported detailed patient characteristics, as well as anatomical, resting-state functional, and diffusion-weighted MRI results (Ceko et al., 2013). Here we report results of an fMRI working memory task ( $N$-back). As reported previously, the groups were matched on years of education, individual annual income, level of physical activity, and menopausal status (for detail, see Ceko et al., 2013). Furthermore, the groups did not differ in terms of estimated intellectual capacity [Wechsler Test of Adult Reading (Pearson Clinical Assessment) scores \pm SD in controls, $43 \pm 5$ and patients, $41 \pm 6 ; p=0.166]$, and there was no indication of malingering in any of the subjects [all subjects passed the Word Memory Test for performance validity (Green et al., 1999)]. Exclusion criteria for all subjects included smoking, use of recreational drugs, use of opioid medication, alcohol consumption of $>10$ United Kingdom units per week, pregnancy or breastfeeding, chronic pain conditions other than fibromyalgia, major medical, neurological, or current psychiatric conditions, including severe depression and generalized anxiety disorder, and MRI contraindications. All patients included in this study had a diagnosis of fibromyalgia and exclusion of other (pain) 
disorders confirmed by an experienced rheumatologist (M.-A.F.). Patients were on stable preventive or as per needed medication (NSAIDs, $n=24$; low-dose antidepressants, $n=13$; muscle relaxants, $n=5$; anticonvulsants, $n=7$; triptans, $n=2$; cannabinoids, $n=1$ ).

All procedures were approved by the McGill University Institutional Review Board, and written informed consent was obtained from all subjects according to the Declaration of Helsinki.

\section{MRI session}

MRI acquisition. FMRI data were not obtained in five healthy subjects (in two subjects, the MRI session was not completed because of time constraints, the MRIs of two subjects had artifacts, and one subject declined participation). In total, 23 healthy subjects and 28 pain patients completed a $10 \mathrm{~min}$ anatomical MRI scan (resolution, $1 \times 1 \times 1 \mathrm{~mm}$; scan details reported by Ceko et al., 2013) and an 8 min fMRI scan during which they performed the widely used $N$-back working memory task. Throughout the session, subjects wore earplugs and their heads were immobilized. Brain images were acquired using a 3 tesla Siemens TIMTrio scanner (Siemens) with a standard 12 channel head coil. fMRI data were acquired using a blood oxygenation level-dependent (BOLD) protocol with a $\mathrm{T} 2{ }^{*}$-weighted gradient echo planar imaging (EPI) sequence (TR, $2260 \mathrm{~ms}$; TE, $30 \mathrm{~ms}$; flip angle, $90^{\circ}$; resolution, $3.5 \times 3.5 \times 3.5 \mathrm{~mm}$; field of view, $224 \mathrm{~mm}$ ). Axial slices were oriented $30^{\circ}$ from the line between the anterior and posterior commissures, covering the entire brain and excluding the eyes. After discarding the first three volumes to allow for steady-state magnetization, 230 volumes were acquired.

Cognitive task. During the fMRI, the working memory $N$-back task with three levels of difficulty and a control condition were administered. In the control condition [0-back $(0 \mathrm{~b})]$, subjects were required to press a button whenever the letter $\mathrm{X}$ appeared. Thus, this condition had attentional demand but no working memory demand (i.e., minimal cognitive load). During the 1-back (1b), 2-back (2b), and 3-back (3b) conditions, subjects were instructed to press the button with their right hand if the currently presented letter was the same as the one presented one, two, or three trials back, respectively. Thus, the cognitive load increased with each task condition. In total, four conditions $(0 \mathrm{~b}, 1 \mathrm{~b}, 2 \mathrm{~b}, 3 \mathrm{~b})$ were presented in 12 pseudorandomized $25 \mathrm{~s}$ blocks (three repeats for each condition, all subjects received the same sequence), with three target letters (X for $0 \mathrm{~b}$, other consonants for the other conditions) per block in a pseudorandom order. Blocks were separated by $14 \mathrm{~s}$ of fixation period (black crosshair on a white background). Each block was preceded by a $3 \mathrm{~s}$ announce period. Each stimulus (letter) was presented for $2 \mathrm{~s}$, with an interstimulus interval of $0.5 \mathrm{~s}$. The reaction times and response accuracy were recorded online. Subjects were familiarized with the $N$-back task before entering the scanner. Most subjects ( 21 of 23 controls and 27 of 28 patients) were right-handed [as confirmed by the Edinburgh Handedness Inventory (Oldfield, 1971)]. Left-handed subjects did not have slower reaction times ( $>1$ SD of group mean) than the right-handed subjects on any task level.

Current pain. To assess possible interference of ongoing pain with cognitive performance and fMRI findings, the intensity of current pain during the fMRI was assessed immediately after the fMRI scan on an 11 point numerical pain intensity rating scale from 0 to 10 ( 0 , no pain; 1 , pain threshold; 10 , worst bearable pain).

\section{Behavioral data analysis}

All data are expressed as means \pm SDs, unless not normally distributed, in which case they are presented as medians and interquartile range (IQR; 25-75\%). Outcome measures were compared between groups in SPSS version 21 (IBM) using repeated-measures (RM) ANOVA or independent samples two-tailed $t$ tests or nonparametric tests in case of nonnormally distributed data, and a significance level of $p<0.05$ was used in all analyses. Correlations between behavioral measures and fMRI data were investigated using Pearson's correlations or Spearman's correlations for non-normally distributed data.

\section{MRI data preprocessing and analysis}

General linear model analysis of N-back $f$ MRI data. Cognitive task fMRI data were preprocessed and analyzed in AFNI (Automated Functional Neuro-Imaging; http://afni.nimh.nih.gov). Briefly, preprocessing in- cluded slice time correction, six parameter (three translations and three rotations) rigid-body correction for head motion, coregistration to the T1-weighted anatomical image, and spatial normalization to MNI space using a 12 parameter affine registration, followed by smoothing with an 8 $\mathrm{mm}$ Gaussian kernel. To test whether there were any group differences in overall head motion that might affect the fMRI analysis, we compared mean total motion [defined as the square root of the sum of squares of the six motion parameters in healthy controls $(0.098 \pm 0.03 \mathrm{~mm})$ and pain patients $(0.110 \pm 0.05 \mathrm{~mm})]$. There was no significant group difference in head motion $(p=0.33)$. Groups also did not differ significantly in the amount of excessive head motion (defined as mean total motion being above an AFNI default value of $0.3 \mathrm{~mm}$ ) with an average of $5.1 \%$ of the time series in healthy controls and $8.6 \%$ in pain patients $(p=0.23)$. Periods (TRs) of excessive motion were excluded from the analysis.

The $N$-back stimuli blocks were modeled at the subject level using AFNI $3 \mathrm{dDeconvolve} \mathrm{regression} \mathrm{with} \mathrm{the} \mathrm{time} \mathrm{courses} \mathrm{of} \mathrm{the} \mathrm{six} \mathrm{motion}$ parameters included as regressors of no interest. A mixed-effects general linear model (GLM) including estimated within-subject variability (AFNI 3dMEMA) was used for group comparisons (two-sample $t$ tests) of $N$-back contrast maps $(1 \mathrm{~b}-0 \mathrm{~b}, 2 \mathrm{~b}-0 \mathrm{~b}, 3 \mathrm{~b}-0 \mathrm{~b})$. Results were considered significant at a voxelwise threshold of $p<0.01$ and were cluster corrected for multiple comparisons $(p<0.05)$ using Monte Carlo simulation (AFNI 3dClustSim) across an independently defined mask of external-task-positive and DMN brain regions. This brain mask comprised regions implicated in fMRI studies of "working memory" (734 studies) and "default mode" (355 studies) as defined using the meta-analytical software Neurosynth (Yarkoni et al., 2011; http://neurosynth.org).

Modulation by load in DMN regions: ROI analysis. In the two regions showing the highest deactivation (most pronounced negative fMRI response) during task across subjects [i.e., the core regions of the DMN (Mayer et al., 2010)] and showing significant group differences (PCC/ precuneus and MPFC), we extracted the fMRI signal in each cluster for each task level and investigated group differences in modulation of fMRI activity by cognitive load (i.e., responsiveness to increasing task difficulty) using an RM-ANOVA in SPSS version 21 (IBM).

Modulation by load in the DMN: multivariate network analysis with partial least squares. Although the PCC/precuneus and MPFC are the core regions of the DMN (Mayer et al., 2010), there are other regions considered to comprise the whole DMN network. Therefore, we performed an additional network analysis of the DMN to investigate the modulation by cognitive load at the network level. We applied the multivariate task partial least squares (PLS) analysis (McIntosh and Lobaugh, 2004; Seminowicz and Davis, 2007) on the preprocessed fMRI data in the DMN mask to assess functionally coactivated patterns of brain activity related to the four task levels $(0 b, 1 b, 2 b, 3 b$, i.e., increasing cognitive load) and to assess groups differences.

PLS detects brain networks showing covariance of BOLD activity with experimental conditions to identify a new set of variables that optimally relate the two ["latent variables" (LVs)]. The PLS analysis is data driven, because contrasts between task levels and groups are not specified in advance. The LVs are ordered by the amount of covariance they explain (similar to a principal component analysis), and the number of latent structures is constrained by the number of experimental conditions (i.e., 4 task levels $\times 2$ groups). Each LV contains a functional map of brain regions that, as a whole, show the strongest relation to the experimental condition. Brain voxels comprising an LV each have a weight (salience) that is proportional to the covariance of BOLD activity with experimental conditions. The "design scores" indicate how strongly and in which direction a given functional map covaries with a certain experimental condition and can be positively or negatively related to the spatial covariance map.

The grand mean deviation PLS method was used (over all subjects and conditions). Data were averaged within and across blocks of the same condition within each subject, so that one data point is used for each condition for each subject. Two statistical tests were performed in PLS. First, the significance of each LV was determined by permutation testing with 500 permutations, yielding a $p$ value for each LV. Second, statistical reliability of task effects was determined by calculating SEs of the saliences for each voxel using bootstrap resampling with 1000 iterations, 
which provided confidence intervals for the LVs, as well as the bootstrap ratio for each voxel [the ratio of the observed saliences to the bootstrapped SE; approximately equivalent to a $\mathrm{Z}$ score and thresholded at 2.3 $(\sim p<0.01)]$.

Compensatory neural recruitment. Adopting methods used in previous studies in which increased activation during cognitive task performance was interpreted to be compensatory, we compared the following between groups: (1) fMRI activation during the task to detect areas with increased activation in patients compared with controls (Glass et al., 2011; Weissman-Fogel et al., 2011) in external-task-positive regions but also elsewhere in the brain (whole-brain gray matter analysis); and (2) the spatial extent of neural recruitment during the task [calculated as the total number of activated voxels above a threshold of $t=2$ for each subject for each task contrast of interest $(1 b-0 b, 2 b-0 b, 3 b-0 b)$ across the gray matter of the whole brain to detect more spatially widespread activation in patients compared with controls (Seminowicz et al., 2011)].

Relationship between responsiveness to increasing task load and cognitive performance. Based on previous research directly linking the responsiveness of external-task-positive regions to increasing cognitive demand (i.e., modulation of fMRI activity by task load) with task performance (Nagel et al., 2009, 2011) notably in frontal cortices, we investigated the correlation between task accuracy on the difficult task level (3b) and the responsiveness to increasing task load in external-task-positive ROI and in the DMN ROIs [in clusters of significant group differences, calculated as responsiveness (fMRI signal change fixation to $3 \mathrm{~b}$ ) - (fMRI signal change fixation to $0 \mathrm{~b}$ ), and capturing the entire slope from minimal to high cognitive load].

Meaning of a less responsive DMN in pain patients. For an alternative meaning of a less responsive DMN in patients, we investigated the relationship between responsiveness (modulation of fMRI activity by increasing task load) in DMN regions and pain coping. fMRI activity in DMN regions has been linked to rumination (Kross et al., 2009) and specifically to increased rumination and catastrophizing about pain in patients with chronic pain (Kucyi et al., 2014), so we examined the relationship between patients' responsiveness to increasing task load in the DMN regions MPFC and PCC and their score on the pain catastrophizing scale (PCS; captures pain-related rumination, helplessness, and magnification; group results reported by Ceko et al., 2013). We also examined the relationship between patients' DMN responsiveness and their current pain.

\section{Results}

Healthy subjects and pain patients performed comparably on the $N$-back task

Both healthy subjects and pain patients performed the control (0b) and $1 \mathrm{~b}$ tasks at $100 \%$ accuracy (median value), with comparable short reaction times ( $\sim 550-660 \mathrm{~ms})$.

As the task became more difficult, accuracy declined and reaction times increased in both groups, consistent with values reported in other studies (Harvey et al., 2005; Nagel et al., 2009; Mayer et al., 2010). Additionally, there was no significant difference between healthy subjects and pain patients in performance or reaction time on the $N$-back task (Fig. 2; Table 1). Therefore, because task performance was similar between groups (pain patients did not perform significantly worse), condition 1 (Fig. 1) was not met.

\section{Despite similar task performance, pain patients showed less deactivation of DMN regions}

Both healthy subjects and pain patients showed deactivation in DMN regions during task performance [deactivation defined as less fMRI signal during working memory task (1b, 2b, or $3 b$ ) compared with the control task 0b]. However, despite similar performance on the task, patients had significantly less deactivation than healthy subjects in the PCC/precuneus and MPFC dur-
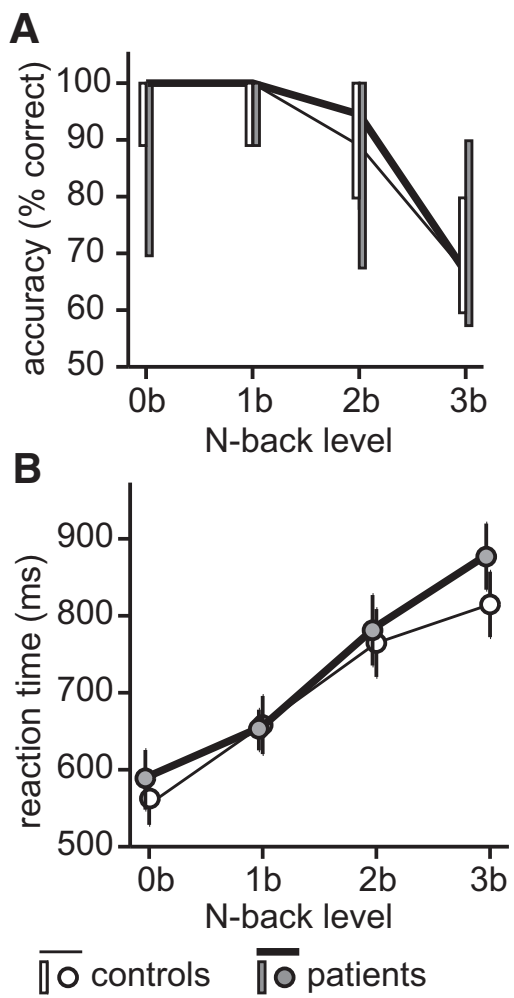

Figure 2. $\quad N$-back task performance. Controls and patients displayed worsening of performance as cognitive load increased (ANOVA, $p<0.001$ ), but groups did not significantly differ in their performance on any of the levels (ANOVA, $p$ values $>0.2$ ). $\boldsymbol{A}$, Task accuracy expressed as percentage correct responses, medians, and IQRs. $\boldsymbol{B}$, Task reaction time in milliseconds, means and SEM. Controls, Thin line, white markers; patients, thick line, gray markers.

\section{Table 1. $N$-back task behavioral performance}

\begin{tabular}{lllll}
\hline & Controls & Patients & $p$ & $p^{\dagger}$ (distribution) \\
\hline $\begin{array}{llll}\text { Accuracy (\%) } \\
\text { median (IQR) }\end{array}$ & & & & \\
Ob & & & & \\
1b & $100(11.0)$ & $100(30.6)$ & $>0.99$ & 0.582 \\
2b & $100(11.0)$ & $100(11.0)$ & $>0.99$ & 0.836 \\
3b & $89.0(12.9)$ & $94.5(33.3)$ & 0.622 & 0.778 \\
Reaction time (ms) & $66.7(22.3)$ & $66.7(33.3)$ & 0.968 & 0.822 \\
mean (SD) & & & & \\
Ob & & & & \\
1b & $553(132)$ & $587(205)$ & 0.550 & \\
2b & $660(186)$ & $652(140)$ & 0.863 & \\
3b & $769(216)$ & $781(242)$ & 0.855 & \\
\hline
\end{tabular}

Patients' current pain ( $2.60 \pm 2.71,18$ of 28 had pain) was not correlated with their performance on the $\mathrm{N}$-back task on any difficulty level (accuracy, $\rho<-0.23, p>0.27$; reaction time, $r<-0.28, p>0.16$ ). Healthy subjects had no pain during the fMRI scan (except 1 individual with a score of 1 of 10 ).

*Equality of variances between groups per task level (homogeneity of variance, $p>0.05$ ) verified by a nonparametric Levene's test (Nordstokke et al., 2011).

${ }^{\dagger}$ Kruskal-Wallis test.

ing the $2 \mathrm{~b}$ (medium) condition (relative to the control condition 0b; Fig. 3A; Table 2). There were no areas in which patients had more pronounced deactivation compared with controls. Similarly, in the $3 \mathrm{~b}$ condition (difficult; highest task load), patients showed less deactivation of the PCC/precuneus, with no areas exhibiting more pronounced deactivation in patients compared with controls. In additional analyses and to ensure that patients did not have altered activation during the control task (0b), we also compared 0b-fixation between groups (no group differences) and each level (1b, 2b, 3b) with fixation (results consistent 

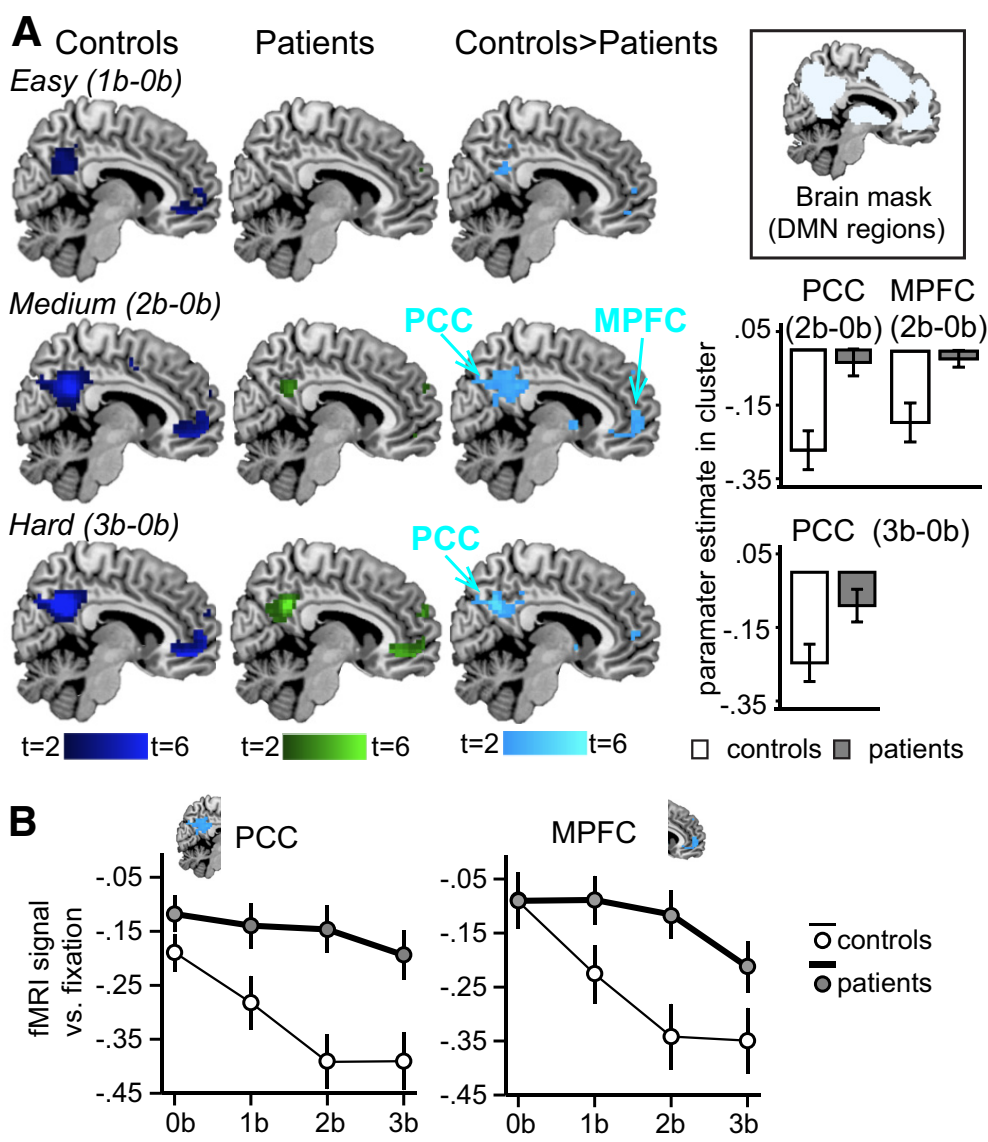

$\overline{\text { C BOLD signal in a representative region (PCC) I controls Ipatients }}$

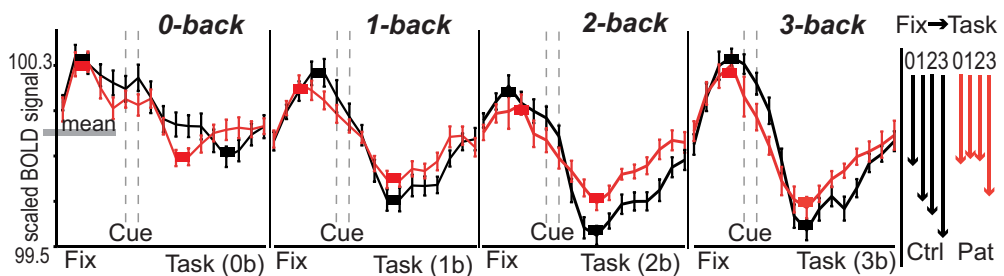

mean-scaled BOLD signal; Fix=Fixation period; Cue=Announce period; Task=Task period
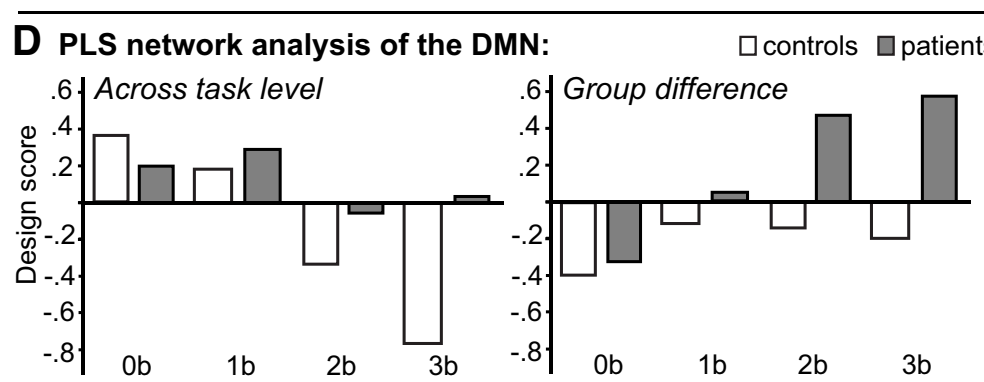

Figure 3. DMN deactivation at different task levels and DMN responsiveness to increasing cognitive load. $\boldsymbol{A}$, Healthy controls showed the usual pattern of deactivation (e.g., negative fMRI response; blue statistical $t$ map) in DMN regions during each level of the $N$-back task compared with the control condition (0b) with minimal cognitive load [easy $(1 b-0 b)$, medium $(2 b-0 b)$, hard $(3 b-0 b)]$. In patients, the deactivation of DMN regions (green) was significantly reduced in the PCC/precuneus and MPFC at $2 b-$ $\mathrm{Ob}$ and in the $\mathrm{PCC} /$ precuneus at $3 \mathrm{~b}-0 \mathrm{~b}$ [pale blue, $p<0.05$ cluster-corrected across an independently defined brain mask consisting of DMN regions (shown here in the inset) and task-positive regions (Fig. 4, inset)]. Color bars are tvalues from 2 to 6 . Bar graphs show the fMRI signal parameter estimates for controls (white) and patients (gray) in significant brain clusters (averaged across each cluster, for $2 \mathrm{~b}-0 \mathrm{~b}$ contrast: PCC/precuneus cluster size, $6646 \mathrm{~mm}^{3}$; MPFC cluster size, $3601 \mathrm{~mm}^{3}$; for $3 \mathrm{~b}-0 \mathrm{~b}$ contrast: $\mathrm{PCC} /$ precuneus cluster size, $5016 \mathrm{~mm}^{3}$ ). Error bars are SEM. B, fMRI signal (scaled and averaged per task level per group, expressed in percentage change relative to fixation) plotted for each task level in the PCC/precuneus cluster (left) and MPFC cluster (right). Groups showed similar deactivation from fixation to $\mathrm{ob}$ (control task, no working memory requirement) but then diverged during task levels with working-memory requirement, so that healthy controls showed increased deactivation in DMN regions with increasing task difficulty [between the control task $0 \mathrm{~b}$ and the difficult task (3b); white circles], and this modulation with comparisons with $0 \mathrm{~b})$. We also examined the shape of the distribution of the task performance (accuracy) across the study sample, and although we could not identify a bimodal distribution on either $2 \mathrm{~b}$ or $3 \mathrm{~b}$, we performed an additional median split analysis to compare the amount of deactivation between "low" and "high" performers and found no significant differences (deactivation low vs high, on 2b: PCC, $p=0.746$; MPFC, $p=$ 0.220 ; on $3 \mathrm{~b}$ : PCC, $p=0.886$ ). We also compared "extremely low" and "extremely high" performers by removing the values around the median and found no significant differences (deactivation extremely low vs extremely high, on $2 \mathrm{~b}$ : PCC, $p=0.699$; MPFC, $p=0.399$; on $3 \mathrm{~b}$ : PCC, $p=0.872$ ).

\section{Pain patients showed impaired} modulation of deactivation by cognitive load (responsiveness) in the DMN

Although healthy subjects showed the commonly observed modulation of deactivations in DMN regions as the cognitive load increased (McKiernan et al., 2003), in patients this modulation of fMRI deactivation by increasing task level was significantly reduced in both DMN clusters (ANOVA on the fMRI signal in the PCC: level, $p<0.001$; group, $p=0.004$; group $\times$ level, $p=0.008$; ANOVA on the

\section{$\leftarrow$}

of the fMRI signal by increasing task load was attenuated significantly in patients (gray circles; group effects per RMANOVA on the fMRI signal in the PCC/precuneus: level, $p<$ 0.001 ; group, $p=0.004$; group $\times$ level, $p=0.008$; ANOVA on the fMRI signal in the MPFC: level, $p<0.001$; group, $p=$ 0.037 ; group $\times$ level, $p=0.011$ ). Error bars are SEM. C, fMRI signal [mean scaled at 100 (arbitrary value), averaged per group] during each level of the task for a representative region [PCC, the cluster that shows most pronounced group differences on the medium $(2 b-0 b)$ and difficult $(3 b-0 b)$ task level]. Please note the degree of deactivation (negative fMRI response) relative to preceding fixation fMRI response at each task level for controls (black line) and patients (red line). For simplicity, we marked the peak of activation (during fixation) and peak of deactivation (during task) for each respective level of the task to illustrate that groups show similar magnitude of deactivation in the control task $0 \mathrm{~b}$ (no working memory load), but controls overall show increasingly more deactivation with increasing working memory load (task levels $1 \mathrm{~b}, 2 \mathrm{~b}$, and $3 \mathrm{~b}$; right, black arrows), although this modulation by level is reduced in patients (right, red arrows; except on 3b, as also seen in $\boldsymbol{B}$ ). Error bars are SEM. $\boldsymbol{D}$, Network analysis (PLS) of the relationship between brain activity and task level. Modulation on network level of $\mathrm{fMRl}$ activity by cognitive load in the DMN is observed across both groups (left, $p<0.014$ ) but is reduced significantly in patients compared with controls (right, $p<$ 0.001). Design scores (arbitrary units) indicate how strongly and in which direction the DMN functional map covaries with increasing task difficulty (0b, 1b, 2b, and 3b). Controls, White bars; patients, gray bars. 
Table 2. $N$-back task fMRI results

\begin{tabular}{|c|c|c|c|c|}
\hline Region & Peak $T$ & $\begin{array}{l}\text { Peak MNI } \\
\text { coordinate }\end{array}$ & $\begin{array}{l}\text { Cluster } \\
\text { volume }\left(\mathrm{mm}^{3}\right)\end{array}$ & $\begin{array}{l}\text { Cluster } \\
p\end{array}$ \\
\hline \multicolumn{5}{|l|}{ Deactivations versus $0 \mathrm{~b}^{*}$} \\
\hline \multicolumn{5}{|l|}{$1 \mathrm{~b}$} \\
\hline \multicolumn{5}{|l|}{ Controls > patients: } \\
\hline \multicolumn{5}{|c|}{ no significant clusters } \\
\hline \multicolumn{5}{|c|}{ Patients > controls: } \\
\hline \multicolumn{5}{|c|}{ no significant clusters } \\
\hline \multicolumn{5}{|l|}{$2 \mathrm{~b}$} \\
\hline \multicolumn{5}{|l|}{ Controls $>$ patients } \\
\hline PCC/precuneus & 4.6 & $-2,-69,37$ & 6646 & $<0.001$ \\
\hline MPFC & 3.8 & $-2,57,-5$ & 3601 & 0.012 \\
\hline \multicolumn{5}{|l|}{ Patients > controls: } \\
\hline \multicolumn{5}{|c|}{ no significant clusters } \\
\hline \multicolumn{5}{|l|}{$3 b$} \\
\hline \multicolumn{5}{|l|}{ Controls $>$ patients } \\
\hline PCC/precuneus & 5.5 & $2,-62,37$ & 5016 & $<0.001$ \\
\hline \multicolumn{5}{|l|}{ Activations versus $0 \mathrm{~b}$} \\
\hline \multicolumn{5}{|l|}{$1 \mathrm{~b}$} \\
\hline \multicolumn{5}{|l|}{ Controls > patients: } \\
\hline \multicolumn{5}{|c|}{ no significant clusters } \\
\hline \multicolumn{5}{|l|}{ Patients > controls: } \\
\hline \multicolumn{5}{|c|}{ no significant clusters } \\
\hline \multicolumn{5}{|l|}{$2 b$} \\
\hline \multicolumn{5}{|l|}{ Controls > patients: } \\
\hline \multicolumn{5}{|c|}{ no significant clusters } \\
\hline \multicolumn{5}{|l|}{ Patients > controls: } \\
\hline \multicolumn{5}{|c|}{ no significant clusters } \\
\hline \multicolumn{5}{|l|}{$3 b$} \\
\hline \multicolumn{5}{|l|}{ Controls $>$ patients } \\
\hline VLPFC & 4.3 & $-47,26,16$ & 2830 & 0.042 \\
\hline Fusiform gyrus $^{\dagger}$ & 6.2 & $-40,-55,-19$ & 3516 & 0.013 \\
\hline Patients $>$ controls & & & & \\
\hline no significant & & & & \\
\hline
\end{tabular}

*0b, Control condition with minimal cognitive load.

${ }^{\dagger}$ Activation in the fusiform gyrus was correlated inversely with the amount of pain patients experienced during the scan $(\rho=-0.498, p=0.008)$. No other activations or deactivations were related to current pain in patients [VLPFC (3b), $\rho=0.196, p=0.595 ; \mathrm{PCC}(3 \mathrm{~b}), \rho=-0.196, p=0.318 ; \mathrm{PCC}(2 \mathrm{~b}), \rho=0.071, p=0.721 ; \mathrm{MPFC}$ (2b), $\rho=0.156, p=0.427]$.

fMRI signal in the MPFC: level, $p<0.001$; group, $p=0.037$; group $\times$ level, $p=0.011$; Fig. $3 B$, also see Fig. $3 C$ in which we display the time course per task level in a representative cluster, the PCC). Modulation of deactivation by task level was significantly different from zero in controls (linear regression testing the null hypothesis that the slope is $0: t=-3.349, p=0.001$ for PCC; $t=-3.726, p<0.001$ for MPFC) but not in patients (linear regression, $t=-1.282, p=0.203$ for $\mathrm{PCC} ; t=-1.868, p=0.064$ for MPFC).

\section{Network analysis of modulation by cognitive load}

We further performed a PLS network analysis of the DMN to assess whether the independent regions PCC/precuneus and MPFC, typically assumed as core regions of the DMN because they often show similar behavior, indeed showed common behavior as part of a network. The PLS analysis revealed two significant LVs (components): ( 1 ) one LV ( $p<0.014$ and explaining $24 \%$ of the variance) showing modulation of fMRI activity by cognitive load across the DMN (strongest in the PCC/precuneus and MPFC); and (2) the other LV ( $p<0.001$ and explaining 59\% of the variance) showing a significant reduction of DMN modulation by task load in patients (Fig. 3D). This multivariate analysis thus adds to the univariate GLM findings in regions associated typically with the DMN, by showing impaired modulation by load in patients on a network level, including the contribution of several other areas, overall indicating that the DMN was functioning as a network rather than independent regions.

Therefore, because pain patients had attenuated deactivation (negative fMRI response) and a decreased modulation of deactivation by increasing task load of the DMN, despite similar task performance, condition 2 (Fig. 1) was not met.

\section{Patients showed no compensatory recruitment of neural resources}

We next investigated whether the pain patients showed increased recruitment of neural resources to suggest compensation for attenuated DMN deactivation, as reported previously in other groups of pain patients (Seminowicz et al., 2011; WeissmanFogel et al., 2011). We first examined the responses of subjects in typical external-task-positive regions, including lateral prefrontal and superior parietal cortices (Fig. 4, inset). Both healthy subjects and pain patients activated the external-task-positive regions on all task levels. No areas of the typical external-task-positive network showed increased activation in patients during the $N$-back task (Fig. 4A) compared with controls. Contrary to having compensatory increases in external-task-positive activations, patients actually had less activation than controls in the ventrolateral prefrontal cortex (VLPFC) and the fusiform gyrus (Fig. 4A, C). In the fusiform gyrus activation decrease was related significantly to the amount of pain patients experienced during the scan (Table 2).

We also performed a whole-brain analysis to determine whether patients had more widespread activation than healthy subjects outside the typical external-task-positive network. No areas showed increased activation in patients compared with controls during any level of the $N$-back task. Furthermore, as shown in Figure $4 B$, patients did not have more total activated voxels across the whole brain on any task level [at $t=2$ as used previously (Seminowicz et al., 2011): $1 \mathrm{~b}, p=0.821 ; 2 \mathrm{~b}, p=0.873$; $3 \mathrm{~b}, p=0.165$; and at a more liberal threshold of $t=1.65: 1 \mathrm{~b}, p=$ $0.805 ; 2 \mathrm{~b}, p=0.656 ; 3 \mathrm{~b}, p=0.130$; and a higher threshold of $t=$ 3 : $1 \mathrm{~b}, p=0.815 ; 2 \mathrm{~b}, p=0.676 ; 3 \mathrm{~b}, p=0.355]$. To ensure that patients did not have increased or more widespread activation at the $0 \mathrm{~b}$ level and this is why no (additional) compensation is seen, we additionally compared the contrast $0 \mathrm{~b}$ to fixation between groups and the number of activated voxels in $1 \mathrm{~b}, 2 \mathrm{~b}$, and $3 \mathrm{~b}$ to fixation and found no group differences. Thus, despite robust overall external-task-related activation, we did not find any evidence for compensatory recruitment of neural resources in patients applying the same methods used in previous studies in which increased activation during cognitive task performance was interpreted to be compensatory. Therefore, because pain patients showed no compensatory recruitment of external-taskpositive regions to maintain equal task performance, condition 3 (Fig. 1) was not met.

\section{Relationship between responsiveness to task load and cognitive performance}

Based on previous research directly linking the responsiveness (i.e., modulation by cognitive load) of several external-taskpositive regions (including VLPFC) to individual variability in cognitive performance (i.e., task accuracy on the most difficult level), we investigated this link in the external-task-positive cluster (VLPFC) and DMN clusters (PCC, MPFC), identified by main effect differences between groups.

Both groups showed significant responsiveness (modulation of fMRI activity by task level) in the VLPFC with no significant 


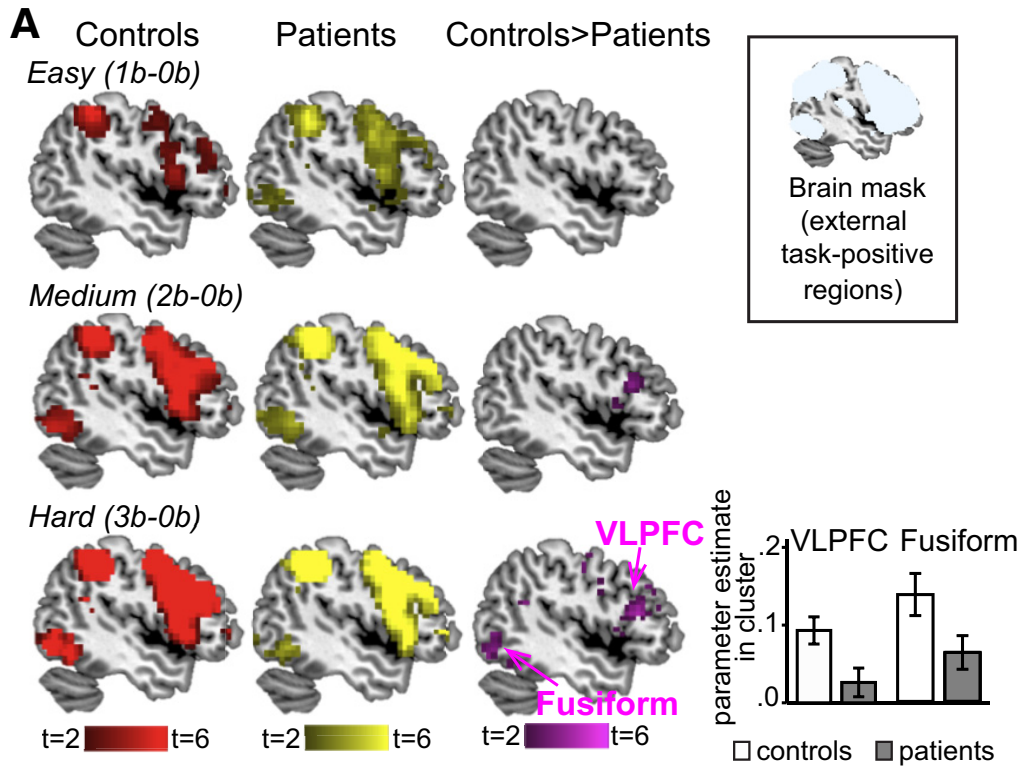

B
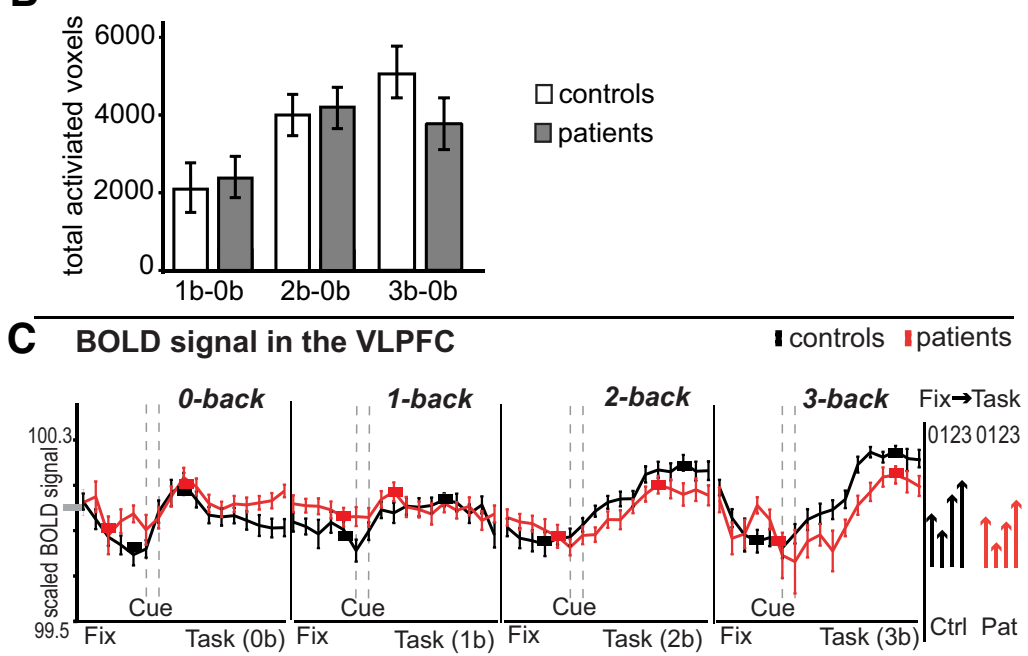

mean-scaled BOLD signal; Fix=Fixation period; Cue=Announce period; Task=Task period

Figure 4. Investigation of compensatory recruitment. $\boldsymbol{A}$, Activation in task-positive regions. There were no areas in which patients (yellow) showed stronger activation than controls (red) at any task level. Patients show decreased activation on $3 \mathrm{~b}-0 \mathrm{~b}$ in the left VLPFC and left fusiform gyrus [purple, controls $>$ patients, $p<0.05$ cluster-corrected across an independently defined brain mask consisting of external-task-positive regions (shown here in the inset) and DMN regions (Fig. 3, inset)]. Color bars are $t$ values from 2 to 6 . Bar graphs show the fMRI signal parameter estimates in significant clusters for controls (white) and patients (gray). Error bars are SEM. B, Spatial distribution of fMRI activity. Patients and controls had comparable spatial distribution of activation on each $N$-back level. Bar graphs show the total number of activated voxels across the whole brain in controls (white) and patients (gray), thresholded at $t>2$. Error bars are SEM. C, fMRI signal [mean scaled at 100 (arbitrary value), averaged per group] during each level of the task for the VLPFC to illustrate that both groups show robust activation of the VLPFC on the $2 \mathrm{~b}$ and $3 \mathrm{~b}$, although this activation is dampened in patients (red line) compared with controls (black line). Error bars are SEM.
PCC/MPFC in controls, $\rho=0.032, p=$ 0.886 ; in patients, $\rho=0.043, p=0.827$. Therefore, although we replicated previous reports of a significant positive relationship between responsiveness of an external-task-positive region (VLPFC) and cognitive performance, we did not observe a relationship between the responsiveness of DMN regions (PCC, MPFC) and cognitive performance.

In an additional exploratory analysis, we also investigated the overall relationship between the spatial extent of fMRI activations (total number of voxels above $t=2$ ) during task levels of interest (2b, 3b) and task performance (accuracy). Across subjects, we observed a significant correlation between the number of activated voxels during task and task accuracy (2b, $\rho=0.401, p=$ 0.004; 3b: $\rho=0.339, p=0.015)$. The total number of deactivated voxels showed no significant correlation with task accuracy on either task level (across subjects: $2 \mathrm{~b}, p=0.591$; $3 \mathrm{~b}, p=0.437$; also investigated for each groups separately because groups differed in the number of deactivated voxels, controls: $2 \mathrm{~b}, p=0.810$; $3 \mathrm{~b}, p=0.573$; patients: $2 \mathrm{~b}, p=0.389$; $3 \mathrm{~b}, p=0.799)$.

\section{Meaning of a less responsive DMN in pain patients}

If the DMN is not linked directly to cognitive performance, why do DMN regions in patients show less responsiveness to increasing cognitive demand in a working memory task? DMN has been implicated in ruminative thinking, including rumination and catastrophizing about pain (Kross et al., 2009; Kucyi et al., 2014). Therefore, we examined the relationship between patients' altered responsiveness to increasing task load in the DMN regions MPFC and PCC and their score on the PCS, as well as their level of current pain. Responsiveness of the MPFC to task load was related positively to PCS scores, so that patients with a less responsive MPFC showed higher pain catastrophgroup difference, albeit of different shape (ANOVA on the fMRI signal: level, $p<0.001$; group, $p=0.421$; level $\times$ group, $p=$ $0.005)$. Across all subjects, responsiveness to increasing cognitive load in the VLPFC was correlated positively with task performance $(\rho=0.446, p=0.001$; Fig. $5 A)$.

In the PCC and in the MPFC, the responsiveness to increasing task load was not related to task accuracy [PCC/MPFC (signal averaged across the two clusters) $\rho=-0.015, p=0.940$; Fig. $5 B$; similar results separately for PCC, $\rho=0.084, p=0.560$; MPFC, $\rho=-0.083, p=0.564]$. Because patients showed significantly reduced responsiveness compared with controls, we also investigated the relationship with accuracy in each group separately: izing $(r=0.391, p=0.044$; Fig. 6$)$. The correlation was not significant for the PCC $(r=0.088, p=0.654)$. Responsiveness of the DMN regions was not significantly related to patients' current pain (MPFC, $\rho=0.018, p=0.929$; PCC, $\rho=-0.278$, $p=0.151)$.

\section{Discussion}

Here we show that a fully responsive DMN may not be required for successful cognitive performance. During a multilevel $N$-back working memory task, despite comparable task performance on each task level, patients showed less deactivation and reduced modulation (responsiveness) of fMRI activity by task load in the 
A
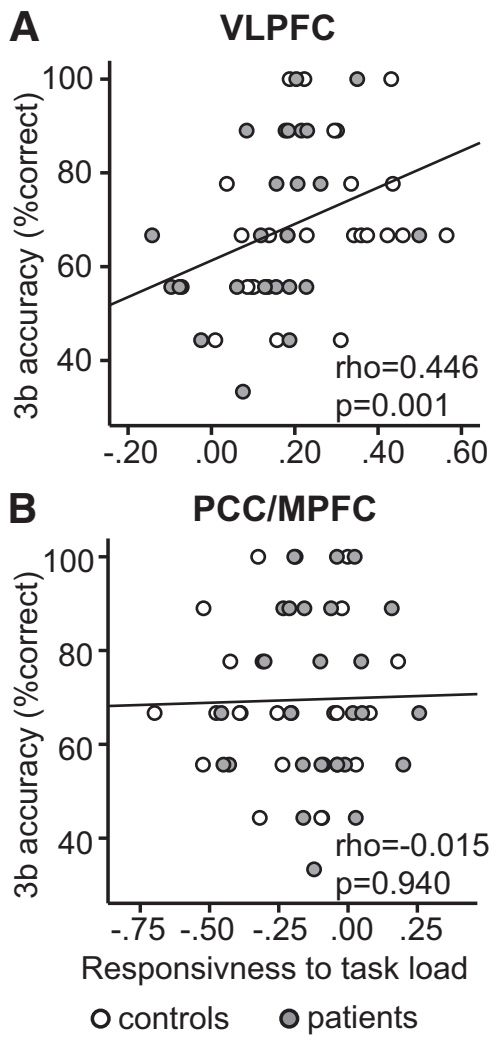

Figure 5. Relationship between responsiveness of external-task-positive and DMN regions to cognitive load and task performance. $A$, Responsiveness to increasing cognitive load in the VLPFC is across subjects significantly correlated with task accuracy on $3 b$. Controls, White circles; patients, gray circles; regression line is across all subjects. $\boldsymbol{B}$, Responsiveness to increasing cognitive load in the PCC/precuneus and MPFC (values averaged across the two ROIs) is across subjects not significantly correlated with task accuracy on $3 \mathrm{~b}$. Responsiveness is calculated as (fMRI signal change from fixation to $3 \mathrm{~b}$ ) - (fMRI signal change from fixation to $0 \mathrm{~b}$ ) to capture the entire slope from minimal to high cognitive load. Controls, White circles; patients, gray circles; regression line is across all subjects.

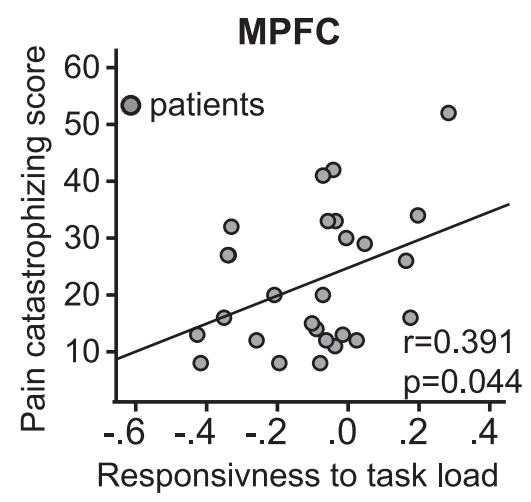

Figure 6. Correlations of DMN responsiveness with pain catastrophizing in pain patients. Responsiveness to increasing cognitive load in the MPFC is in patients significantly correlated with their score on the PCS. Responsiveness is calculated as (fMRI signal change fixation to 3b) - (fMRI signal change fixation to $0 \mathrm{~b}$ ) to capture the entire slope from minimal to high cognitive load. Patients, Gray circles; regression line across patients.

DMN, without any observable compensatory recruitment of neural resources.

\section{Attenuated responsiveness of the DMN to increasing cognitive demand in pain patients}

Across all $N$-back levels, patients displayed similar task performance (accuracy) as controls and similar reaction times, indicat- ing similar decision time. Despite successful performance, patients had less deactivation (negative fMRI response) of DMN regions, as reported previously in chronic pain patients (Baliki et al., 2008; Seminowicz et al., 2011; Weissman-Fogel et al., 2011). We extend previous findings by showing that $\mathrm{DMN}$ regions also were significantly less responsive (i.e., had less modulation of the negative fMRI response with increasing task load), as well as showing that DMN acted as a network rather than individual regions. The responsiveness of DMN regions to increasing task load was not correlated with task performance in patients or controls. Typically in paradigms with varying task difficulty, load-dependent modulation (responsiveness) of deactivation is observed in DMN regions (Shulman et al., 1997; McKiernan et al., 2003; Todd et al., 2005; Mayer et al., 2010), as we also show here in controls. This responsiveness to increasing cognitive demand has been interpreted as the necessary reallocation of finite cognitive resources to external-task-positive processes needed for cognitive performance (Sambataro et al., 2010; Anticevic et al., 2012a; Metzak et al., 2012). Increased cognitive demand is suggested to be reflected in increased neural processing in externaltask-positive regions involved in working memory and attention (Braver et al., 1997; Tomasi et al., 2007; Rottschy et al., 2012) and correspondingly in an increased reallocation of cognitive resources toward external-task-positive processes. Thus, it has been suggested that modulation of deactivation (responsiveness) to increasing task load in the DMN is functionally significant, because it facilitates successful cognitive performance (Kim et al., 2010; Anticevic et al., 2012a). Our results suggest that successful cognitive performance can be achieved despite reduced modulation (responsiveness) of the DMN to increasing cognitive demand. Nevertheless, our patients did show a small amount of DMN modulation. It is possible that there is a threshold amount of DMN deactivation or modulation that is necessary and sufficient to maintain task performance (Esterman et al., 2013, 2014), with our patients having reached that threshold. Another less functional population (e.g., dementia) might not reach that threshold for DMN modulation, with resulting poor performance.

The significance of responsive external-task-positive activations for successful working memory functioning In the external-task-positive VLPFC, the responsiveness of fMRI activity to increasing cognitive load was related directly to task performance, such that those individuals with the most responsive VLPFC to increasing cognitive load had the highest accuracy on the most difficult level of the $\mathrm{N}$-back task. This finding replicates previous reports directly linking working memory performance to neural responsiveness in several external-task-positive regions, including the VLPFC (Nagel et al., 2009, 2011; Burzynska et al., 2011), supporting the notion that the responsiveness of external-task-positive activations to increasing task demand is needed for successful cognitive functioning. The VLPFC might be particularly important in this context, because it shows load dependence across working memory studies (Rottschy et al., 2012). Studies have also shown that individual variability in the amount of activation of external-task-positive regions is related to successful task performance (Rypma et al., 2007; Yarkoni et al., 2009; Gess et al., 2014; Rao et al., 2014).

The relationship of task-related responsiveness to load and task-related deactivations to cognitive task performance Evidence linking modulation of deactivation by increasing task load (responsiveness) in DMN regions with successful working memory performance is lacking, and in fact, we ob- 
serve no significant relationship between the two in the current study.

Moreover, the evidence linking individual variability in the amount of deactivation in DMN regions during a cognitive task to successful cognitive performance is mixed. Although a relationship between impaired deactivation of the core DMN regions and impaired working memory performance has been reported, especially in schizophrenia and in aging (Whitfield-Gabrieli et al., 2009), several recent studies in healthy individuals reported no significant relationship between DMN regional deactivation and task performance during memory and attention tasks (Gess et al., 2014; Rao et al., 2014). The amount of deactivation in the PCC and the temporoparietal junction during memory encoding has been linked to successful subsequent memory retrieval (Daselaar et al., 2004; Anticevic et al., 2010, 2012a). Decreased activation in DMN regions, most notably the PCC/precuneus, during memory encoding could support the relative increase (vs encoding period) in activation sometimes observed in these regions during subsequent memory performance and during (episodic) memory retrieval (Andrews-Hanna et al., 2010a; Spreng et al., 2010; Anticevic et al., 2012b). Therefore, increased deactivation in the DMN could play a role in successful encoding of items into memory for later retrieval, more so than in "executive" working memory processes during tasks such as the $\mathrm{N}$-back in which cognitive resources possibly undergo continuous reallocation.

\section{Attenuated deactivation and compensatory neural recruitment}

Similar to our results, less deactivation in DMN regions during a cognitive task has been observed in a number of disorders, including chronic pain, despite patients showing similar task performance as controls. Typically, however, these studies show activation patterns in patients that have been interpreted as compensatory neural recruitment to maintain equal performance. In chronic pain patients performing cognitive tasks of similar difficulty to the task used here, more activation of external-taskpositive regions (Weissman-Fogel et al., 2011) or a larger number of activated voxels across the brain (Seminowicz et al., 2011) were interpreted as compensation for attenuated deactivation in DMN regions. These mechanisms are not consistent across pain studies but have together with increased activations in external-taskpositive regions observed in other disorders and in aging (Harvey et al., 2005; Sambataro et al., 2010; Metzak et al., 2012) provided evidence for compensatory recruitment of cortical resources. We found no evidence for compensation using the same methods. Because we tested for the presence of compensation in a relatively large sample [28 patients compared with 18 patients (Seminowicz et al., 2011) and 17 patients (Weissman-Fogel et al., 2011)], it is unlikely that we did not have the sensitivity to detect group differences. Because we found no compensatory measures to counter the alterations observed in the DMN applying methods used in previous studies, our findings suggest that a responsive DMN may not be required for successful cognitive performance.

\section{What is the significance of reduced DMN responsiveness?}

If decreased responsiveness of DMN regions to increasing task demand does not negatively affect cognitive performance, what is the significance of this reduced responsiveness in patients? We observed a positive relationship between reduced MPFC responsiveness and pain catastrophizing, suggesting that, in patients, the DMN could, during the cognitive task, be partly engaged in excessive thinking about pain, manifesting as decreased loaddependent modulation of deactivation. Self-referential thinking and mind wandering has been linked to DMN activity (Kross et al., 2009; Kucyi et al., 2013). Thus, rumination about pain in patients with high pain catastrophizing scores might serve as a "mind wandering-like" distraction from the task and result in some residual DMN activation with a net effect of attenuated DMN deactivation. Indeed, pain catastrophizing and rumination have been linked to DMN activity, including in the MPFC, in chronic pain patients (Kucyi et al., 2014). Thus, our findings support the role of DMN in some forms of self-related processing. Pain patients with fibromyalgia often experience significant subjective cognitive difficulties ("fibrofog"). Although this study was not able to explore the relationship between fibrofog and diminished DMN responsiveness, this could be investigated in a future study.

\section{Alternative explanations and study limitations}

An alternative explanation for the observed fMRI findings could be that the patients' brain activity during the task was dampened and less responsive overall. For regions showing increased activation during the task, this is unlikely, because patients showed robust activation (similar to controls) across the external-taskpositive network (albeit reduced on $3 \mathrm{~b}$ ), as well as modulation of neural activity by cognitive load. Nevertheless, decreased activation on the $3 \mathrm{~b}$ might potentially account for decreased deactivation (i.e., generally dampened response on the highest task level), which might serve to partially preserve the balance between the external-task-positive network and DMN. We did not observe a significant group difference using a proxy measure of balance between networks (calculated as the ratio of VLPFC activation/ deactivation in either PCC or MPFC, $p>0.2$ on each task level), suggesting that a preserved balance between cognitive networks, which is likely relevant for cognitive processing (Kelly et al., 2008), might aid task performance in patients.

It could be that the manipulation of task difficulty was not strong enough to modulate DMN deactivations (Shulman et al., 1997). However, this is unlikely in our study, because we did observe a significant modulation by load in controls in both external-task-positive and $\mathrm{DMN}$ regions.

Because we tested only females in this study, this could preclude a generalization of our findings to males. However, recent work has shown that females and males have similar task performance and task-related brain activity during the $N$-back task (Schmidt et al., 2009), and, in fact, it has been observed that intra-gender differences are larger than inter-gender differences across several cognitive domains (Hyde, 2005).

Finally, use of medications such as anticonvulsants could have in several patients contributed to decreased recruitment and possibly also decreased responsiveness of brain regions during the cognitive task (Beltramini et al., 2015).

\section{Conclusion}

Our study extends previous findings in chronic pain patients by showing not only altered deactivation in DMN regions but also reduced modulation of deactivations (responsiveness) to increasing task load in the DMN. Nevertheless, patients maintained similar task performance as controls and this apparently without any compensatory neural recruitment. We observed a positive correlation between the responsiveness of the external-task-positive VLPFC and task performance but no relationship between the responsiveness of DMN regions and task performance. Thus, although we provide additional support for the importance of responsive external-task-positive regions for cognitive functioning, we conclude that the responsiveness of the DMN to increasing 
cognitive demand may not be required for successful working memory performance.

\section{References}

Andrews-Hanna JR, Reidler JS, Huang C, Buckner RL (2010a) Evidence for the default network's role in spontaneous cognition. J Neurophysiol 104: 322-335. CrossRef Medline

Andrews-Hanna JR, Reidler JS, Sepulcre J, Poulin R, Buckner RL (2010b) Functional-anatomic fractionation of the brain's default network. Neuron 65:550-562. CrossRef Medline

Anticevic A, Repovs G, Shulman GL, Barch DM (2010) When less is more: TPJ and default network deactivation during encoding predicts working memory performance. Neuroimage 49:2638-2648. CrossRef Medline

Anticevic A, Cole MW, Murray JD, Corlett PR, Wang XJ, Krystal JH (2012a) The role of default network deactivation in cognition and disease. Trends Cogn Sci 16:584-592. CrossRef Medline

Anticevic A, Gancsos M, Murray JD, Repovs G, Driesen NR, Ennis DJ, Niciu MJ, Morgan PT, Surti TS, Bloch MH, Ramani R, Smith MA, Wang XJ, Krystal JH, Corlett PR (2012b) NMDA receptor function in large-scale anticorrelated neural systems with implications for cognition and schizophrenia. Proc Natl Acad Sci U S A 109:16720-16725. CrossRef Medline

Baliki MN, Geha PY, Apkarian AV, Chialvo DR (2008) Beyond feeling: chronic pain hurts the brain, disrupting the default-mode network dynamics. J Neurosci 28:1398-1403. CrossRef Medline

Barch DM, Braver TS, Nystrom LE, Forman SD, Noll DC, Cohen JD (1997) Dissociating working memory from task difficulty in human prefrontal cortex. Neuropsychologia 35:1373-1380. CrossRef Medline

Beltramini GC, Cendes F, Yasuda CL (2015) The effects of antiepileptic drugs on cognitive functional magnetic resonance imaging. Quant Imaging Med Surg 5:238-246. CrossRef Medline

Braver TS, Cohen JD, Nystrom LE, Jonides J, Smith EE, Noll DC (1997) A parametric study of prefrontal cortex involvement in human working memory. Neuroimage 5:49-62. CrossRef Medline

Burzynska AZ, Nagel IE, Preuschhof C, Li SC, Lindenberger U, Bäckman L, Heekeren HR (2011) Microstructure of frontoparietal connections predicts cortical responsivity and working memory performance. Cereb Cortex 21:2261-2271. CrossRef Medline

Ceko M, Bushnell MC, Fitzcharles MA, Schweinhardt P (2013) Fibromyalgia interacts with age to change the brain. Neuroimage Clin 3:249-260. CrossRef Medline

Christoff K, Gordon AM, Smallwood J, Smith R, Schooler JW (2009) Experience sampling during fMRI reveals default network and executive system contributions to mind wandering. Proc Natl Acad Sci U S A 106: 8719-8724. CrossRef Medline

Daselaar SM, Prince SE, Cabeza R (2004) When less means more: deactivations during encoding that predict subsequent memory. Neuroimage 23: 921-927. CrossRef Medline

Esterman M, Noonan SK, Rosenberg M, Degutis J (2013) In the zone or zoning out? Tracking behavioral and neural fluctuations during sustained attention. Cereb Cortex 23:2712-2723. CrossRef Medline

Esterman M, Rosenberg MD, Noonan SK (2014) Intrinsic fluctuations in sustained attention and distractor processing. J Neurosci 34:1724-1730. CrossRef Medline

Fox MD, Snyder AZ, Vincent JL, Corbetta M, Van Essen DC, Raichle ME (2005) The human brain is intrinsically organized into dynamic, anticorrelated functional networks. Proc Natl Acad Sci U S A 102:9673-9678. CrossRef Medline

Fransson P (2005) Spontaneous low-frequency BOLD signal fluctuations: an fMRI investigation of the resting-state default mode of brain function hypothesis. Hum Brain Mapp 26:15-29. CrossRef Medline

Gess JL, Fausett JS, Kearney-Ramos TE, Kilts CD, James GA (2014) Taskdependent recruitment of intrinsic brain networks reflects normative variance in cognition. Brain Behav 4:650-664. CrossRef Medline

Glass JM, Williams DA, Fernandez-Sanchez ML, Kairys A, Barjola P, Heitzeg MM, Clauw DJ, Schmidt-Wilcke T (2011) Executive function in chronic pain patients and healthy controls: different cortical activation during response inhibition in fibromyalgia. J Pain 12:1219-1229. CrossRef Medline

Green P, Iverson GL, Allen L (1999) Detecting malingering in head injury litigation with the Word Memory Test. Brain Inj 13:813-819. CrossRef Medline

Gusnard DA, Akbudak E, Shulman GL, Raichle ME (2001) Medial prefron- tal cortex and self-referential mental activity: relation to a default mode of brain function. Proc Natl Acad Sci U S A 98:4259-4264. CrossRef Medline

Harvey PO, Fossati P, Pochon JB, Levy R, Lebastard G, Lehéricy S, Allilaire JF, Dubois B (2005) Cognitive control and brain resources in major depression: an fMRI study using the n-back task. Neuroimage 26:860-869. CrossRef Medline

Hyde JS (2005) The gender similarities hypothesis. Am Psychol 60:581-592. CrossRef Medline

Kelly AM, Uddin LQ, Biswal BB, Castellanos FX, Milham MP (2008) Competition between functional brain networks mediates behavioral variability. Neuroimage 39:527-537. CrossRef Medline

Kim H, Daselaar SM, Cabeza R (2010) Overlapping brain activity between episodic memory encoding and retrieval: roles of the task-positive and task-negative networks. Neuroimage 49:1045-1054. CrossRef Medline

Kross E, Davidson M, Weber J, Ochsner K (2009) Coping with emotions past: the neural bases of regulating affect associated with negative autobiographical memories. Biol Psychiatry 65:361-366. CrossRef Medline

Kucyi A, Salomons TV, Davis KD (2013) Mind wandering away from pain dynamically engages antinociceptive and default mode brain networks. Proc Natl Acad Sci U S A 110:18692-18697. CrossRef Medline

Kucyi A, Moayedi M, Weissman-Fogel I, Goldberg MB, Freeman BV, Tenenbaum HC, Davis KD (2014) Enhanced medial prefrontal-default mode network functional connectivity in chronic pain and its association with pain rumination. J Neurosci 34:3969-3975. CrossRef Medline

Mayer JS, Roebroeck A, Maurer K, Linden DE (2010) Specialization in the default mode: task-induced brain deactivations dissociate between visual working memory and attention. Hum Brain Mapp 31:126-139. CrossRef Medline

McIntosh AR, Lobaugh NJ (2004) Partial least squares analysis of neuroimaging data: applications and advances. Neuroimage 23 [Suppl 1]:S250S263. CrossRef

McKiernan KA, Kaufman JN, Kucera-Thompson J, Binder JR (2003) A parametric manipulation of factors affecting task-induced deactivation in functional neuroimaging. J Cogn Neurosci 15:394-408. CrossRef Medline

McKiernan KA, D'Angelo BR, Kaufman JN, Binder JR (2006) Interrupting the "stream of consciousness": an fMRI investigation. Neuroimage 29: 1185-1191. CrossRef Medline

Metzak PD, Riley JD, Wang L, Whitman JC, Ngan ET, Woodward TS (2012) Decreased efficiency of task-positive and task-negative networks during working memory in schizophrenia. Schizophr Bull 38:803-813. CrossRef Medline

Nagel IE, Preuschhof C, Li SC, Nyberg L, Bäckman L, Lindenberger U, Heekeren HR (2009) Performance level modulates adult age differences in brain activation during spatial working memory. Proc Natl Acad Sci U S A 106:22552-22557. CrossRef Medline

Nagel IE, Preuschhof C, Li SC, Nyberg L, Bäckman L, Lindenberger U, Heekeren HR (2011) Load modulation of BOLD response and connectivity predicts working memory performance in younger and older adults. J Cogn Neurosci 23:2030-2045. CrossRef Medline

Nordstokke DW, Zumbo BD, Cairns SL, Safklofske DH (2011) The operating characteristics of the nonparametric Levene test for equal variances with assessment and evaluation data. Pract Assess Res Eval 16:5.

Oldfield RC (1971) The assessment and analysis of handedness: the Edinburgh inventory. Neuropsychologia 9:97-113. CrossRef Medline

Raichle ME, MacLeod AM, Snyder AZ, Powers WJ, Gusnard DA, Shulman GL (2001) A default mode of brain function. Proc Natl Acad Sci U S A 98:676-682. CrossRef Medline

Rao NK, Motes MA, Rypma B (2014) Investigating the neural bases for intra-subject cognitive efficiency changes using functional magnetic resonance imaging. Front Hum Neurosci 8:840. CrossRef Medline

Rottschy C, Langner R, Dogan I, Reetz K, Laird AR, Schulz JB, Fox PT, Eickhoff SB (2012) Modelling neural correlates of working memory: a coordinate-based meta-analysis. Neuroimage 60:830-846. CrossRef Medline

Rypma B, Eldreth DA, Rebbechi D (2007) Age-related differences in activation-performance relations in delayed-response tasks: a multiple component analysis. Cortex 43:65-76. CrossRef Medline

Sambataro F, Murty VP, Callicott JH, Tan HY, Das S, Weinberger DR, Mattay VS (2010) Age-related alterations in default mode network: impact on 
working memory performance. Neurobiol Aging 31:839-852. CrossRef Medline

Schmidt H, Jogia J, Fast K, Christodoulou T, Haldane M, Kumari V, Frangou S (2009) No gender differences in brain activation during the N-back task: an fMRI study in healthy individuals. Hum Brain Mapp 30:36093615. CrossRef Medline

Seminowicz DA, Davis KD (2007) Pain enhances functional connectivity of a brain network evoked by performance of a cognitive task. J Neurophysiol 97:3651-3659. CrossRef Medline

Seminowicz DA, Wideman TH, Naso L, Hatami-Khoroushahi Z, Fallatah S, Ware MA, Jarzem P, Bushnell MC, Shir Y, Ouellet JA, Stone LS (2011) Effective treatment of chronic low back pain in humans reverses abnormal brain anatomy and function. J Neurosci 31:7540-7550. CrossRef Medline

Shulman GL, Fiez JA, Corbetta M, Buckner RL, Miezin FM, Raichle ME, Petersen SE (1997) Common blood flow changes across visual tasks: II. Decreases in cerebral cortex. J Cogn Neurosci 9:648-663. CrossRef Medline

Spreng RN, Stevens WD, Chamberlain JP, Gilmore AW, Schacter DL (2010) Default network activity, coupled with the frontoparietal control network, supports goal-directed cognition. Neuroimage 53:303-317. CrossRef Medline

Todd JJ, Fougnie D, Marois R (2005) Visual short-term memory load sup- presses temporo-parietal junction activity and induces inattentional blindness. Psychol Sci 16:965-972. CrossRef Medline

Tomasi D, Chang L, Caparelli EC, Ernst T (2007) Different activation patterns for working memory load and visual attention load. Brain Res 1132: 158-165. CrossRef Medline

Weissman-Fogel I, Moayedi M, Tenenbaum HC, Goldberg MB, Freeman BV, Davis KD (2011) Abnormal cortical activity in patients with temporomandibular disorder evoked by cognitive and emotional tasks. Pain 152: 384-396. CrossRef Medline

Whitfield-Gabrieli S, Thermenos HW, Milanovic S, Tsuang MT, Faraone SV, McCarley RW, Shenton ME, Green AI, Nieto-Castanon A, LaViolette P, Wojcik J, Gabrieli JD, Seidman LJ (2009) Hyperactivity and hyperconnectivity of the default network in schizophrenia and in first-degree relatives of persons with schizophrenia. Proc Natl Acad Sci U S A 106: 1279-1284. CrossRef Medline

Yarkoni T, Barch DM, Gray JR, Conturo TE, Braver TS (2009) BOLD correlates of trial-by-trial reaction time variability in gray and white matter: a multi-study fMRI analysis. PLoS One 4:e4257. CrossRef Medline

Yarkoni T, Poldrack RA, Nichols TE, Van Essen DC, Wager TD (2011) Large-scale automated synthesis of human functional neuroimaging data. Nat Methods 8:665-670. CrossRef Medline 